\title{
Spacecraft Attitude Tracking and Maneuver Using Combined Magnetic Actuators
}

\author{
Zhiqiang Zhou ${ }^{1}$ \\ NASA Langley Research Center, Hampton, VA 23681
}

The accuracy of spacecraft attitude control using magnetic actuators only is low and on the order of 0.4-5 degrees. The key reason is that the magnetic torque is two-dimensional and it is only in the plane perpendicular to the magnetic field vector. In this paper novel attitude control algorithms using the combination of magnetic actuators with Reaction Wheel Assembles (RWAs) or other types of actuators, such as thrusters, are presented. The combination of magnetic actuators with one or two RWAs aligned with different body axis expands the two-dimensional control torque to three-dimensional. The algorithms can guarantee the spacecraft attitude and rates to track the commanded attitude precisely. A design example is presented for Nadir pointing, pitch and yaw maneuvers. The results show that precise attitude tracking can be reached and the attitude control accuracy is comparable with RWAs based attitude control. The algorithms are also useful for the RWAs based attitude control. When there are only one or two workable RWAs due to RWA failures, the attitude control system can switch to the control algorithms for the combined magnetic actuators with the RWAs without going to the safe mode and the control accuracy can be maintained.

\section{Nomenclature}

$\begin{array}{ll}\tilde{b}(t) & =\text { Earth's magnetic field vector } \\ \hat{D}(t), \hat{K}(t) & =\text { control gain matrices } \\ J & =\text { inertia matrix } \\ m & =\text { vector of magnetic dipoles } \\ \delta \bar{q} & =\text { attitude error quaternion } \\ \bar{q} & =\text { quaternion } \\ T & =\text { control torque } \\ \omega & =\text { angular velocity } \\ \Omega(\omega) & =\text { skew-symmetric matrix }\end{array}$

\section{Introduction}

Magnetic torque bars as attitude control actuators have been used and studied for many years. The typical uses of magnetic actuators in attitude control are for Reaction Wheel Assembles (RWAs) de-saturation, and angularmomentum and nutation control of spinning and momentum-biased spacecraft. There are some studies Ref.1-6 to use the magnetic torque bars only to control the spacecraft attitude. Global stable attitude control laws have been investigated for both inertial and earth pointing in Ref.1-2. Periodic control algorithms have been developed using an asymptotic linear quadratic regulator technique for a Nadir-pointing Ref.3. A pseudospectral control law has been applied to the magnetic attitude control of satellites in elliptic low earth orbits in Ref. 4.

The main drawback of the magnetic actuator only attitude control is that the control accuracy is low and on the order of 0.4-5 degrees. The key reason is that the magnetic torque is two-dimensional and it is only in the plane perpendicular to the magnetic field vector. The spacecraft cannot be controlled precisely in three-dimensional space using only magnetic actuators. In order to improve the control accuracy, control algorithms for a combination of

${ }^{1}$ Aerospace Technologist, NASA Langley Research Center, M/S 488, 5 N. Dryden St., Hampton, VA 23681. zhiqiang.zhou@nasa.gov, Senior Member AIAA. 
magnetic actuators with RWAs or other types of actuators are presented in this paper. The combination of magnetic actuators with one or two RWAs aligned with different body axis expands the two-dimensional control torque to three-dimensional. The attitude control accuracy for the combined actuators using the control algorithms proposed in this paper is comparable with RWAs based attitude control.

The control algorithms of the combined actuators are also useful for the RWAs based precise attitude control. When there are only two or one workable RWAs due to RWA failures, the attitude control system can switch to the control algorithms for the combined magnetic actuators with the RWAs presented in this paper without going to the safe mode. The control accuracy can be maintained.

This paper is organized as follows. Section II presents the spacecraft dynamics, the kinematics equations, and the control matrix produced by the magnetic actuators. The controllability of magnetic actuators only is investigated. The necessity of the combination of magnetic actuators with RWAs to control the spacecraft attitude precisely is revealed. The combined control matrices with one RWA along different body axis are derived in Section III. The attitude control of magnetic actuators with two RWAs along different body axis is discussed in Section IV. Attitude tracking algorithms are described in Section V and a design example is given in Section VI to demonstrate the performance of the control system.

\section{Problem Statement}

The spacecraft dynamics in the spacecraft body frame can be expressed as

$$
\begin{aligned}
J \dot{\omega} & =-\omega \times(J \omega)+T_{M T B}+T_{R W A}+T_{\text {dist }} \\
& =\Omega(\omega) J \omega+T_{M T B}+T_{R W A}+T_{\text {dist }}
\end{aligned}
$$

where $J$ is the inertia matrix. $\omega=\left(\omega_{x}, \omega_{y}, \omega_{z}\right)^{T} \in \mathrm{R}^{3}$ is the angular velocity with respect to (wrt) an inertial frame expressed in the body frame. $T_{M T B}$ and $T_{R W A}$ are the MTB and RWA control torques. $T_{\text {dist }}$ is the external disturbances torque due to gravity gradient, aerodynamic force, and solar pressure. $\Omega(\omega)$ is a skewsymmetric matrix defined by

$$
\Omega(\omega) \equiv\left(\begin{array}{ccc}
0 & \omega_{z} & -\omega_{y} \\
-\omega_{z} & 0 & \omega_{x} \\
\omega_{y} & -\omega_{x} & 0
\end{array}\right)
$$

Using quaternions, the kinematic equation of motion can be described by

$$
\left\{\begin{array}{l}
\dot{q}=\frac{1}{2} \Omega(\omega) q+\frac{1}{2} q_{4} \omega \\
\dot{q}_{4}=-\frac{1}{2} \omega^{T} q
\end{array} .\right.
$$

The spacecraft attitude is determined by its quaternion $\bar{q}$, which represents the attitude of the spacecraft wrt the inertial frame. Both $q$ and $q_{4}$ of the quaternion $\bar{q}$ are defined as

$$
\begin{gathered}
q \equiv\left(\begin{array}{l}
q_{1} \\
q_{2} \\
q_{3}
\end{array}\right) \equiv\left(\begin{array}{l}
e_{1} \sin (\phi / 2) \\
e_{2} \sin (\phi / 2) \\
e_{3} \sin (\phi / 2)
\end{array}\right) \\
q_{4} \equiv \cos (\phi / 2) \\
\bar{q} \equiv\left(\begin{array}{c}
q \\
q_{4}
\end{array}\right) .
\end{gathered}
$$

In Equation (4), $e_{1}, e_{2}$, and $e_{3}$ are the components of the rotation axis unit vector expressed in the inertial frame; $\phi$ is the rotation angle. 
A set of three magnetic coils aligned with the body frame axes generates the magnetic attitude control torque, which can be written as

$$
T_{\text {MTB }}=m \times \widetilde{b}(t)=\Omega(\widetilde{b}(t)) m
$$

where $m=\left(m_{x}, \quad m_{y}, \quad m_{z}\right)^{T} \in \mathrm{R}^{3}$ is the vector of magnetic dipoles for the three coils. $\tilde{b}(t) \in \mathrm{R}^{3}$ is the Earth's magnetic field vector expressed in the body frame, which is changing all the time and depends on the spacecraft position and attitude. Note that the magnitude of $\widetilde{b}(t)$ is not zero. The following unit vector in the same direction of $\tilde{b}(t)$ is introduced as

$$
b(t)=\frac{\widetilde{b}(t)}{\|\widetilde{b}(t)\|}=\left(b_{x}(t), \quad b_{y}(t), \quad b_{z}(t)\right)^{T} .
$$

Obviously the unit vector $b(t)$ satisfies the following equation

$$
b_{x}^{2}(t)+b_{y}^{2}(t)+b_{z}^{2}(t)=1 .
$$

Consider following form of the vector of magnetic dipoles

$$
m=\frac{1}{\|\tilde{b}(t)\|^{2}} \Omega^{T}(\tilde{b}(t)) u
$$

where $u=\left(u_{x}, u_{y}, u_{z}\right)^{T} \in R^{3}$ is a new control vector. The magnetic control torque $T_{M T B}$ for the magnetic dipoles is

where

$$
\begin{aligned}
T_{\text {MTB }} & =\Omega(b(t)) \Omega^{T}(b(t)) u \\
& =\Gamma(b(t)) u
\end{aligned}
$$

$$
\begin{aligned}
\Gamma(b(t)) & =\Omega(b(t)) \Omega^{T}(b(t)) \\
& =\left(\begin{array}{lll}
b_{y}^{2}(t)+b_{z}^{2}(t) & -b_{x}(t) b_{y}(t) & -b_{x}(t) b_{z}(t) \\
-b_{x}(t) b_{y}(t) & b_{x}^{2}(t)+b_{z}^{2}(t) & -b_{y}(t) b_{z}(t) \\
-b_{x}(t) b_{z}(t) & -b_{y}(t) b_{z}(t) & b_{x}^{2}(t)+b_{y}^{2}(t)
\end{array}\right) .
\end{aligned}
$$

We call $\Gamma(b(t))$ the magnetic control matrix. The control capability and spacecraft dynamic behavior using magnetic actuators are determined by $\Gamma(b(t))$. The following are some properties of $\Gamma(b(t))$.

- $\quad \Gamma(b(t))$ is symmetric and semi-positive definite matrix. It is easy to show the determinant of $\Gamma(b(t))$ is zero.

$$
\operatorname{det}|\Gamma(b(t))|=0
$$

- $\quad \Gamma(b(t))$ always has one zero eigenvalue and two positive real eigenvalues. The eigenvector corresponding to the zero eigenvalue is $b(t)$ given by

$$
\Gamma(b(t)) b(t)=0 .
$$

- If $T_{M T B_{\text {desired }}}$ is the desired magnetic control torque, there is a control vector $u$ only if $T_{M T B_{\text {desired }}}$ is perpendicular to $b(t)$. Therefore, in general, if $T_{M T B_{\text {desired }}}$ is not perpendicular to $b(t)$, there is no $u$ to realize the desired magnetic control torque.

The properties of the magnetic control matrix show that the magnetic control torque $T_{M T B}$ is always in the plane, which is perpendicular to $b(t)$. The magnetic control torque is only two-dimensional. In order to expand the control to three-dimensional, additional actuators need to be added, which can be thrusters or RWAs. In following RWAs are considered, but the analysis and principals are applicable to the thrusters. 


\section{Combination Of Magnetic Actuators With One RWA}

If one RWA is employed, there are three configurations, in which the wheel can be aligned with body frame $x$, or $y$, or $z$ axis.

\section{Configuration \#1: RWA along body $x$ axis}

The control torque $T_{R W A}$ contributed by the RWA along body $x$ axis is given by

$$
T_{R W A}=\left(u_{x}, 0,0\right)^{T} .
$$

The combined control torque from the magnetic actuator and the RWA can be written as

$$
\begin{aligned}
T_{M T B}+T_{R W A} & =\Gamma(b(t)) u+\left(u_{x}, \quad 0, \quad 0\right)^{T} \\
& =\Lambda_{x}(b(t)) u
\end{aligned}
$$

where

$$
\begin{aligned}
\Lambda_{x}(b(t)) & =\left(\Gamma(b(t))+\left(\begin{array}{lll}
1 & 0 & 0 \\
0 & 0 & 0 \\
0 & 0 & 0
\end{array}\right)\right) u \\
& =\left(\begin{array}{ccc}
1+b_{y}^{2}(t)+b_{z}^{2}(t) & -b_{x}(t) b_{y}(t) & -b_{x}(t) b_{z}(t) \\
-b_{x}(t) b_{y}(t) & b_{x}^{2}(t)+b_{z}^{2}(t) & -b_{y}(t) b_{z}(t) \\
-b_{x}(t) b_{z}(t) & -b_{y}(t) b_{z}(t) & b_{x}^{2}(t)+b_{y}^{2}(t)
\end{array}\right)
\end{aligned}
$$

$\Lambda_{x}(b(t))$ is the combined control matrix with RWA along body $x$ axis.

\section{Configuration \#2: RWA along body $y$ axis}

For the RWA along body $y$ axis the combined control torque is given by

$$
\begin{aligned}
T_{M T B}+T_{R W A} & =\Gamma(b(t)) u+\left(\begin{array}{lll}
0, & u_{y}, & 0
\end{array}\right)^{T} \\
& =\Lambda_{y}(b(t)) u
\end{aligned}
$$

where

$$
\Lambda_{y}(b(t))=\left(\begin{array}{ccc}
b_{y}^{2}(t)+b_{z}^{2}(t) & -b_{x}(t) b_{y}(t) & -b_{x}(t) b_{z}(t) \\
-b_{x}(t) b_{y}(t) & 1+b_{x}^{2}(t)+b_{z}^{2}(t) & -b_{y}(t) b_{z}(t) \\
-b_{x}(t) b_{z}(t) & -b_{y}(t) b_{z}(t) & b_{x}^{2}(t)+b_{y}^{2}(t)
\end{array}\right)
$$

Configuration \#3: RWA along body $z$ axis

Similar to other two configurations, the combined control torque can be written as

$$
\begin{gathered}
T_{M T B}+T_{R W A}=\Gamma(b(t)) u+\left(\begin{array}{lll}
0, & 0, & u_{z}
\end{array}\right)^{T} \\
=\Lambda_{z}(b(t)) u \\
\Lambda_{z}(b(t))=\left(\begin{array}{ccc}
b_{y}^{2}(t)+b_{z}^{2}(t) & -b_{x}(t) b_{y}(t) & -b_{x}(t) b_{z}(t) \\
-b_{x}(t) b_{y}(t) & b_{x}^{2}(t)+b_{z}^{2}(t) & -b_{y}(t) b_{z}(t) \\
-b_{x}(t) b_{z}(t) & -b_{y}(t) b_{z}(t) & 1+b_{x}^{2}(t)+b_{y}^{2}(t)
\end{array}\right) .
\end{gathered}
$$

The combined control matrixes $\Lambda_{i}(b(t)) \quad(i=x$, or $y$, or $z)$ representing the RWA aligned with body frame $x$, or $y$, or $z$ axis have the following properties.

- The combined control matrixes are symmetric and the determinants are given by

$$
\operatorname{det}\left|\Lambda_{i}(b(t))\right|=b_{i}^{2}(t) \quad(i=x \text {, or } y, \text { or } z) .
$$


- If $b_{i}(t) \neq 0 \quad(i=x$, or $y$, or $z), \quad \Lambda_{i}(b(t)) \quad(i=x$, or $y$, or $z)$ always has three positive real eigenvalues and an inverse. The control torque provided by $\Lambda_{i}(b(t)) u \quad(i=x$, or $y$, or $z)$ is threedimensional and the spacecraft is fully controlled.

- If $b_{i}(t)=0 \quad(i=x$, or $y$, or $z), \quad \Lambda_{i}(b(t)) \quad(i=x$, or $y$, or $z)$ has two positive real eigenvalues and one zero eigenvalue. The eigenvector corresponding to the zero eigenvalue is $b(t)$. At the same time $b(t)$ is perpendicular to the wheel direction.

For the combination of the magnetic actuators with one wheel the combined control matrixes $\Lambda_{i}(b(t)) \quad(i=x$, or $y$, or $z)$ is three-dimensional control except $b_{i}(t)=0 \quad(i=x$, or $y$, or $z)$, in which it is two-dimensional control.

If the wheel is not aligned with any body frame axis, the combined control matrix should have the same properties because the wheel control torque can be transformed to body frame axis by a direction cosine matrix.

\section{Combination Of Magnetic Actuators With Two RWAs}

If two RWAs are combined with the magnetic actuators, there are three configurations of two wheels aligned with the body frame axis as follows.

\section{Configuration \#4: RWAs along body $x$ and $y$ axes}

The control torque $T_{R W A}$ contributed by the RWAs is given by

$$
T_{R W A}=\left(u_{x}, u_{y}, 0\right)^{T} .
$$

The combined control torque from the magnetic actuator and the RWAs can be written as

where

$$
\begin{aligned}
T_{M T B}+T_{R W A} & =\Gamma(b(t)) u+\left(u_{x}, \quad u_{y}, \quad 0\right)^{T} \\
& =\hat{\Lambda}_{z}(b(t)) u
\end{aligned}
$$

$$
\begin{aligned}
\hat{\Lambda}_{z}(b(t)) & =\left(\Gamma(b(t))+\left(\begin{array}{lll}
1 & 0 & 0 \\
0 & 1 & 0 \\
0 & 0 & 0
\end{array}\right)\right) u \\
& =\left(\begin{array}{ccc}
1+b_{y}^{2}(t)+b_{z}^{2}(t) & -b_{x}(t) b_{y}(t) & -b_{x}(t) b_{z}(t) \\
-b_{x}(t) b_{y}(t) & 1+b_{x}^{2}(t)+b_{z}^{2}(t) & -b_{y}(t) b_{z}(t) \\
-b_{x}(t) b_{z}(t) & -b_{y}(t) b_{z}(t) & b_{x}^{2}(t)+b_{y}^{2}(t)
\end{array}\right) .
\end{aligned}
$$

\section{Configuration \#5: RWAs along body $y$ and $z$ axes}

For the RWAs along body $y$ and $z$ axes the combined control torque is given by

where

$$
\begin{aligned}
T_{M T B}+T_{R W A} & =\Gamma(b(t)) u+\left(0, \quad u_{y}, \quad u_{z}\right)^{T} \\
& =\hat{\Lambda}_{x}(b(t)) u
\end{aligned}
$$

$$
\hat{\Lambda}_{x}(b(t))=\left(\begin{array}{ccc}
b_{y}^{2}(t)+b_{z}^{2}(t) & -b_{x}(t) b_{y}(t) & -b_{x}(t) b_{z}(t) \\
-b_{x}(t) b_{y}(t) & 1+b_{x}^{2}(t)+b_{z}^{2}(t) & -b_{y}(t) b_{z}(t) \\
-b_{x}(t) b_{z}(t) & -b_{y}(t) b_{z}(t) & 1+b_{x}^{2}(t)+b_{y}^{2}(t)
\end{array}\right)
$$


Configuration \#6: RWAs along body $x$ and $z$ axes

Similarly, for the RWAs along body $x$ and $z$ axes the combined control torque can be written as

$$
\begin{aligned}
T_{M T B}+T_{R W A} & =\Gamma(b(t)) u+\left(u_{x}, \quad 0, \quad u_{z}\right)^{T} \\
& =\hat{\Lambda}_{y}(b(t)) u
\end{aligned}
$$

where

$$
\hat{\Lambda}_{y}(b(t))=\left(\begin{array}{ccc}
1+b_{y}^{2}(t)+b_{z}^{2}(t) & -b_{x}(t) b_{y}(t) & -b_{x}(t) b_{z}(t) \\
-b_{x}(t) b_{y}(t) & b_{x}^{2}(t)+b_{z}^{2}(t) & -b_{y}(t) b_{z}(t) \\
-b_{x}(t) b_{z}(t) & -b_{y}(t) b_{z}(t) & 1+b_{x}^{2}(t)+b_{y}^{2}(t)
\end{array}\right) .
$$

The combined control matrixes $\hat{\Lambda}_{i}(b(t)) \quad(i=x$, or $y$, or $z)$ have the following properties.

- The combined control matrixes are symmetric and the determinants are given by

$$
\operatorname{det}\left|\hat{\Lambda}_{i}(b(t))\right|=2\left(1-b_{i}^{2}(t)\right) \quad(i=x, \text { or } y \text {, or } z) .
$$

- If $\left\|b_{i}(t)\right\| \neq 1 \quad(i=x$, or $y$, or $z), \quad \hat{\Lambda}_{i}(b(t)) \quad(i=x$, or $y$, or $z)$ always has three positive real eigenvalues and an inverse. The control torque provided by $\hat{\Lambda}_{i}(b(t)) u \quad(i=x$, or $y$, or $z)$ is threedimensional and the spacecraft is fully controlled.

- If $\left\|b_{i}(t)\right\|=1 \quad(i=x$, or $y$, or $z), \hat{\Lambda}_{i}(b(t)) \quad(i=x$, or $y$, or $z)$ has two positive real eigenvalues and one zero eigenvalue. The eigenvector corresponding to the zero eigenvalue is $b(t)$. At the same time $b(t)$ is perpendicular to both of the two wheel directions. The combined control matrixes are two-dimensional control.
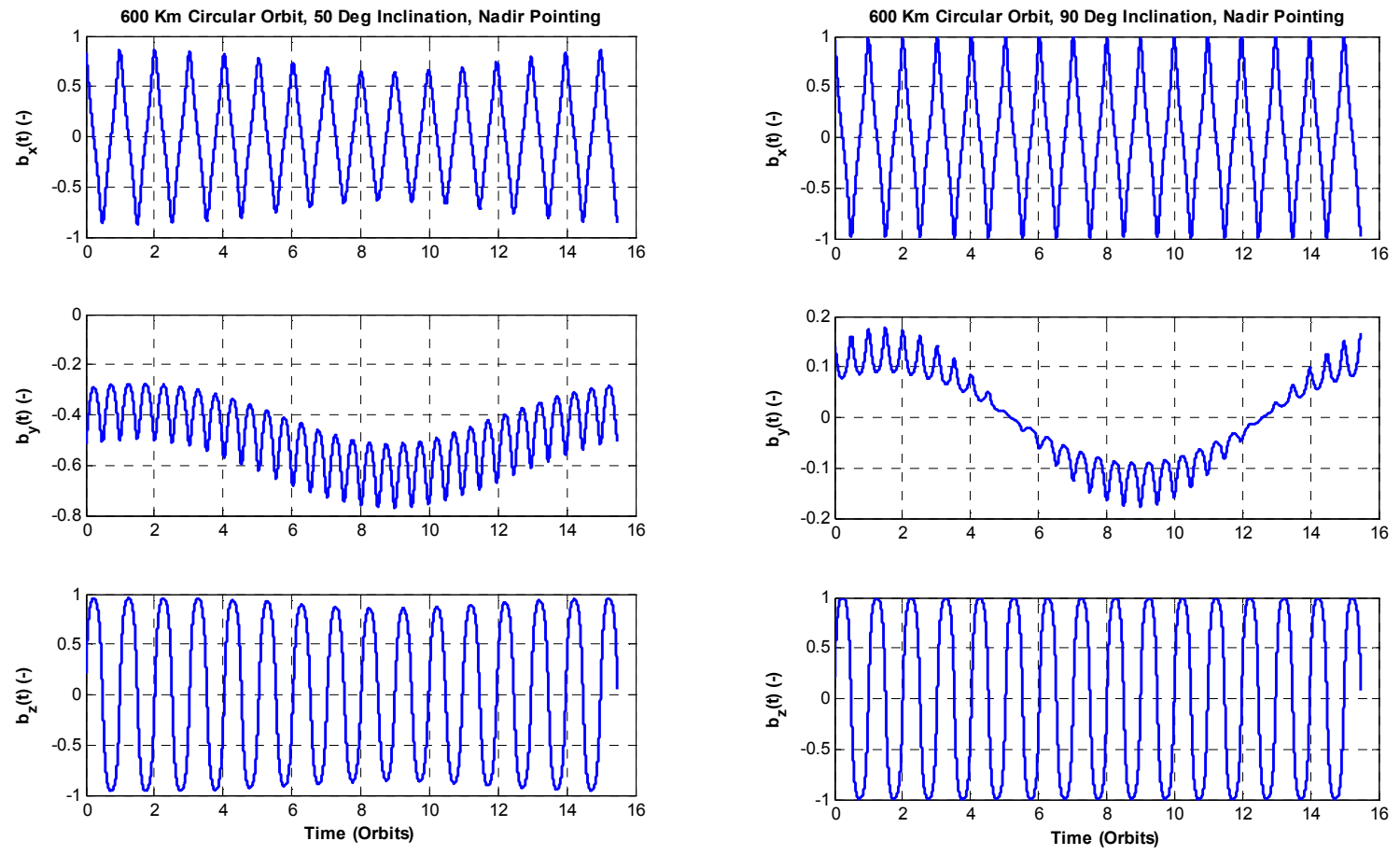

Figure $1 b(t)$ with $600 \mathrm{~km}$ circular orbit and 50 degrees inclination

Figure $2 b(t)$ with $600 \mathrm{~km}$ circular orbit and 90 degrees inclination 
Unlike the combination of the magnetic actuators with one wheel, the combined control matrixes with two wheels are deteriorated to two-dimensional control only if $b(t)$ is in the direction, which is perpendicular to both of the two wheel directions. For two wheels the chance for two-dimensional control is reduced to $b(t)$ along one direction in three-dimensional space from $b(t)$ in a plane, but additional wheel is required as the costs. In practice $b(t)$ is always changing the direction along the spacecraft orbit. There are some orbits and attitudes, in which $b(t)$ does not cross the plane of $b_{i}(t)=0 \quad(i=x$, or $y$, or $z)$ for one wheel combination configurations. Such an orbit is shown in Figure 1, which is a $600 \mathrm{~km}$ circular orbit with 50 degrees inclination. The spacecraft attitude is Nadir pointing. The calculation of $b(t)$ is based on the dipole model in Appendix H of the book Wertz (1978).

As shown in Figure $1, b_{y}(t)$ is not equal to zero for the Nadir pointing spacecraft on the orbit. If the wheel is aligned with body $y$ axis, $\Lambda_{y}(b(t))$ always has an inverse. If the wheel is aligned with body $x$ or $z$ axes, $b_{x}(t)$ or $b_{z}(t)$ will cross zero periodically. $\Lambda_{x}(b(t))$ or $\Lambda_{z}(b(t))$ has no inverse when $b_{x}(t)$ or $b_{z}(t)$ crosses zero.

Figure 2 shows $b(t)$ for a $600 \mathrm{~km}$ circular orbit with 90 degrees inclination and Nadir pointing. All components of $b(t)$ cross zero periodically. $b_{y}(t)$ crosses zero at much slower rate (about every 8 orbits). However, for two wheels configurations, if the two wheels are aligned with $x$ and $z$ axes, $\hat{\Lambda}_{y}(b(t))$ always has an inverse for both orbits because $\left\|b_{y}(t)\right\|$ is always smaller than one.

\section{Attitude Tracking Algorithms and Performance}

For the combination of magnetic actuators with one RWA the spacecraft dynamics (1) becomes

$$
J \dot{\omega}=\Omega(\omega) J \omega+\Lambda_{i}(b(t)) u \quad(i=x, \text { or } y, \text { or } z)
$$

Suppose $\omega_{c}$ and $q_{c}$ are the commanded attitude rate and the commanded vector part of the attitude quaternion to be tracked. Then $\omega_{c}$ is the rotation rate of the command frame with respect to (wrt) an inertial frame resolved in the command frame. Therefore, $\omega_{c}$ and $q_{c}$ satisfy the following kinematic equation of motion

$$
\left\{\begin{array}{l}
\dot{q}_{c}=\frac{1}{2} \Omega\left(\omega_{c}\right) q_{c}+\frac{1}{2} q_{4 c} \omega_{c} \\
\dot{q}_{4 c}=-\frac{1}{2} \omega_{c}^{T} q_{c}
\end{array}\right.
$$

where

$$
\begin{gathered}
\omega_{c} \equiv\left(\begin{array}{lll}
\omega_{x c}, & \omega_{y c}, & \omega_{z c}
\end{array}\right)^{T} \\
\Omega\left(\omega_{c}\right) \equiv\left(\begin{array}{ccc}
0 & \omega_{z c} & -\omega_{y c} \\
-\omega_{z c} & 0 & \omega_{x c} \\
\omega_{y c} & -\omega_{x c} & 0
\end{array}\right) \\
q_{c} \equiv\left(q_{1 c}, \quad q_{2 c}, \quad q_{3 c}\right)^{T}, \quad \bar{q}_{c} \equiv\left(\begin{array}{ll}
q_{c} & q_{4 c}
\end{array}\right)^{T}
\end{gathered}
$$

In order to introduce the control algorithm, the attitude error quaternion $\delta \bar{q}$ needs to be defined as follows: 


$$
\begin{aligned}
\delta \bar{q} & \equiv\left(\begin{array}{c}
\delta q \\
\delta q_{4}
\end{array}\right) \equiv\left(\bar{q}_{c}^{-1} \cdot \bar{q}\right) \\
& =\left(\begin{array}{cccc}
q_{4 c} & q_{3 c} & -q_{2 c} & -q_{1 c} \\
-q_{3 c} & q_{4 c} & q_{1 c} & -q_{2 c} \\
q_{2 c} & -q_{1 c} & q_{4 c} & -q_{3 c} \\
q_{1 c} & q_{2 c} & q_{3 c} & q_{4 c}
\end{array}\right)\left(\begin{array}{l}
q_{1} \\
q_{2} \\
q_{3} \\
q_{4}
\end{array}\right)
\end{aligned}
$$

where $\bar{q}_{c}^{-1}$ is the inverse of $\bar{q}_{c}$, and $\left(\bar{q}_{c}^{-1} \cdot \bar{q}\right)$ is quaternion multiplication. Note that $\delta \bar{q}$ is still a quaternion, which has a physical meaning. If the spacecraft rotates with $\bar{q}_{c}$ and then rotates $\delta \bar{q}$ further, its attitude is the same as if it rotates with $\bar{q}$. Therefore, $\delta \bar{q}$ represents the attitude difference between $\bar{q}$ and $\bar{q}_{c}$. Using the attitude error quaternion, the control algorithm for the combination of magnetic actuators with one RWA is written as follows:

$$
u=-\Lambda_{i}^{-1}(b(t))\left[\Omega(\omega) J \omega+D\left(\omega-\omega_{c}\right)+K \delta q-J \dot{\omega}_{c}\right] \quad(i=x \text {, or } y, \text { or } z)
$$

where $D$ and $K$ are $3 \times 3$ constant gain matrices to be determined. At the orbit point, where $b_{i}(t)=0 \quad(i=x$, or $y$, or $z), \Lambda_{i}(b(t)) \quad(i=x$, or $y$, or $z)$ has no inverse, the inverse at the neighbor orbit points is used to form the control law in (37). Substituting (37) into (31) gives

$$
J\left(\dot{\omega}-\dot{\omega}_{c}\right)+D\left(\omega-\omega_{c}\right)+K \delta q=0 .
$$

Equations (38) and (3) form the closed-loop nonlinear dynamic system of the spacecraft attitude with the control law (37). Using dimensionless control gain matrices, Equation (38) can be rewritten as

$$
\left(\dot{\omega}-\dot{\omega}_{c}\right)+\bar{D}\left(\omega-\omega_{c}\right)+\bar{K} \delta q=0
$$

where

$$
\begin{aligned}
& \bar{D}=J^{-1} D \\
& \bar{K}=J^{-1} K
\end{aligned}
$$

It is not difficult to verify that the following is one of the solutions to Equations (38) and (3)

$$
\left\{\begin{array}{l}
\omega=\omega_{c} \\
q=q_{c}
\end{array}\right.
$$

However, solution (42) is not an equilibrium point of the system because, in general, $\omega$ and $q$ are not constant and are the function of time. When $\omega_{c}=0$ (which implies $q_{c}=$ constant), $\omega=\omega_{c}=0$ and $q=q_{c}=$ constant , solution (42) is an equilibrium point of the system. Solution (42) means that it is possible for the spacecraft to achieve the commanded attitude and attitude rate. This control law can be employed for attitude tracking under any large and fast maneuver because there is no restriction on $\omega_{c}$ and $q_{c}$.

When $\omega_{c} \neq 0$, (42) will not be the solution of the closed-loop system if the term $\Omega(\omega) J \omega$ or $J \dot{\omega}_{c}$ is not included in the control law (37). The stability analysis will not draw any conclusion concerning the spacecraft's ability to track the commanded attitude. For the control law (37) without the term $J \dot{\omega}_{c}$, the solution (42) exists only under condition $\dot{\omega}_{c}=0\left(\omega_{c}=\right.$ constant $)$. This means that the control system can only track the commanded attitude when the attitude rate is constant. If $\omega_{c}=0$ (a rest-to-rest maneuver), the solution (42) exists for the control law (37) without the terms $\Omega(\omega) J \omega, D \omega_{c}$, and $J \dot{\omega}_{c}$.

If the solution (42) has local asymptotic stability, $\omega$ and $q$ (with initial values near the solution) will converge to the solution. If the solution (42) has global asymptotic stability, any $\omega$ and $q$ will converge to the solution. The following theorem shows how to find the constant gain matrices $D$ and $K$ having the globally asymptotic stable solution (42). If such a solution exists, the spacecraft can track the commanded attitude with any commanded attitude rate. 
Theorem Suppose that $\bar{K}^{-1}$ is symmetric and positive definite, and $\bar{K}^{-1} \bar{D}$ is positive definite. $b_{i}(t) \neq 0 \quad(i=x$, or $y$, or $z)$. Then, the control law (37) ensures that the solution (42) is global asymptotically stable and all trajectories $\omega$ and $q$ for the closed-loop nonlinear system (38) and (3) converge to $\omega_{c}$ and $q_{c}$ as time approaches infinity.

The proof of the theorem can be found in the paper Zhou and Colgren (2005a). The magnetic dipoles vector $m$ required by the control law (37) is given by

$$
m=-\frac{1}{\|\tilde{b}(t)\|^{2}} \Omega^{T}(\tilde{b}(t)) \Lambda_{i}^{-1}(b(t))\left[\Omega(\omega) J \omega+D\left(\omega-\omega_{c}\right)+K \delta q-J \dot{\omega}_{c}\right] \quad(i=x, \text { or } y, \text { or } z) .
$$

The corresponding wheel torque is

$$
T_{R W A}= \begin{cases}\left(\begin{array}{lll}
u_{x}, & 0, & 0
\end{array}\right)^{T} & \text { (wheel along } x \text { axis) } \\
\left(\begin{array}{lll}
0, & u_{y}, & 0
\end{array}\right)^{T} & \text { (wheel along } y \text { axis) } \\
\left(\begin{array}{lll}
0, & 0, & u_{z}
\end{array}\right)^{T} & \text { (wheel along } z \text { axis) }\end{cases}
$$

Control law (37) can be rewritten as

$$
u=\hat{D}(t)\left(\omega-\omega_{c}\right)+\hat{K}(t) \delta q-\Lambda_{i}^{-1}(b(t)) \Omega(\omega) J \omega+\Lambda_{i}^{-1}(b(t)) J \dot{\omega}_{c} \quad(i=x, \text { or } y, \text { or } z)
$$

where

$$
\begin{aligned}
& \hat{D}(t)=-\Lambda_{i}^{-1}(b(t)) D \\
& \hat{K}(t)=-\Lambda_{i}^{-1}(b(t)) K
\end{aligned}
$$

The total control gain matrices $\hat{D}(t)$ and $\hat{K}(t)$ are time varying. For two RWAs control law (37) becomes

$$
u=-\hat{\Lambda}_{i}^{-1}(b(t))\left[\Omega(\omega) J \omega+D\left(\omega-\omega_{c}\right)+K \delta q-J \dot{\omega}_{c}\right] \quad(i=x \text {, or } y, \text { or } z) .
$$

Equations (38) and (3) form the closed-loop nonlinear dynamic system of the spacecraft attitude with the control law (48). Similar to the Theorem, if $\bar{K}^{-1}$ is symmetric and positive definite, and $\bar{K}^{-1} \bar{D}$ is positive definite. $\left\|b_{i}(t)\right\| \neq 1 \quad(i=x$, or $y$, or $z)$. Then, the control law (48) ensures that the solution (42) is global stable and all trajectories $\omega$ and $q$ for the closed-loop nonlinear system (38) and (3) converge to $\omega_{c}$ and $q_{c}$ as time approaches infinity. The RWAs torques are given by

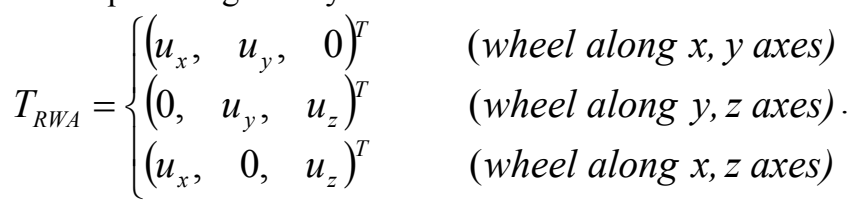

The magnetic dipoles vector $m$ required by the control law (48) is

$$
m=-\frac{1}{\|\widetilde{b}(t)\|^{2}} \Omega^{T}(\widetilde{b}(t)) \hat{\Lambda}_{i}^{-1}(b(t))\left[\Omega(\omega) J \omega+D\left(\omega-\omega_{c}\right)+K \delta q-J \dot{\omega}_{c}\right] \quad(i=x, \text { or } y, \text { or } z) .
$$

To evaluate the performance of the control laws, 3-2-1 Euler angle rotation, yaw $\delta \psi$, pitch $\delta \theta$, and roll $\delta \phi$ corresponding to $\delta \bar{q}$ are employed. Their relations are given by

$$
\left\{\begin{array}{l}
\delta q_{1}= \pm(\sin (\delta \phi / 2) \cos (\delta \theta / 2) \cos (\delta \psi / 2)-\cos (\delta \phi / 2) \sin (\delta \theta / 2) \sin (\delta \psi / 2)) \\
\delta q_{2}= \pm(\cos (\delta \phi / 2) \sin (\delta \theta / 2) \cos (\delta \psi / 2)+\sin (\delta \phi / 2) \cos (\delta \theta / 2) \sin (\delta \psi / 2)) \\
\delta q_{3}= \pm(\cos (\delta \phi / 2) \cos (\delta \theta / 2) \sin (\delta \psi / 2)-\sin (\delta \phi / 2) \sin (\delta \theta / 2) \cos (\delta \psi / 2)) \\
\delta q_{4}= \pm(\cos (\delta \phi / 2) \cos (\delta \theta / 2) \cos (\delta \psi / 2)+\sin (\delta \phi / 2) \sin (\delta \theta / 2) \sin (\delta \psi / 2))
\end{array}\right.
$$

When the Euler angles are small, they can be approximated by 


$$
\left\{\begin{array}{l}
\delta \phi \cong 2 \delta q_{1} \\
\delta \theta \cong 2 \delta q_{2} \\
\delta \psi \cong 2 \delta q_{3}
\end{array}\right.
$$

The attitude rate error $\Delta \omega$ is given by

$$
\Delta \omega=\omega-\omega_{c}
$$

\section{Design Example}

In order to assess the performance of the control law discussed in this paper, a spacecraft attitude control design and simulation tool has been developed. The tool has the following functions:

- Command Generation: generate Nadir pointing, pitch, and yaw maneuver commands

- Controller: control algorithm proposed in this paper

- Spacecraft Dynamic Model: spacecraft rigid body dynamics and orbit mechanics model

- Environmental Models: aero density model (Jacchia Robert), gravity gradient model, geomagnetic model (B field), Solar pressure model

- Solar Ephemeris Model

The considered spacecraft has following inertia matrix

$$
J=\left(\begin{array}{rrr}
288.3053 & 43.8208 & -7.6332 \\
43.8208 & 101.1218 & 0.4111 \\
-7.6332 & 0.4111 & 371.3937
\end{array}\right) \quad\left(\mathrm{Kg} \cdot \mathrm{m}^{2}\right)
$$

It has a solar array with a $1.2758 \mathrm{~m}^{2}$ surface along body y axis. It flies on a $600 \mathrm{~km}$ circular orbit with 50 degrees inclination. Two configurations of wheel alignments are presented here. The first one is that the wheel is aligned with body $y$ axis, in which $\Lambda_{y}(b(t))$ always has an inverse for the Nadir pointing spacecraft on the orbit as shown in Figure 1. For the second configuration the wheel is aligned with body $z$ axis, $b_{z}(t)$ will cross zero periodically and $\Lambda_{z}(b(t))$ has no inverse when $b_{z}(t)$ crosses zero. For both configurations the gain matrices $D$ and $K$ in the control law are selected as

$$
\begin{aligned}
D & =\left(\begin{array}{rrr}
12.8071 & 1.9466 & -0.3391 \\
1.9466 & 4.4920 & 0.0183 \\
-0.3391 & 0.0183 & 16.4981
\end{array}\right) \quad\left(\frac{\mathrm{Kg} \cdot \mathrm{m}^{2}}{\mathrm{~s}}\right) \\
K & =\left(\begin{array}{rrr}
0.5691 & 0.0865 & -0.0151 \\
0.0865 & 0.1996 & 0.0008 \\
-0.0151 & 0.0008 & 0.7331
\end{array}\right) \quad\left(\frac{\mathrm{Kg} \cdot \mathrm{m}^{2}}{\mathrm{~s}^{2}}\right) .
\end{aligned}
$$

$\bar{K}^{-1}$ and $\bar{K}^{-1} \bar{D}$ have the following corresponding values

$$
\begin{gathered}
\bar{K}^{-1}=\left(\begin{array}{rrr}
506.6059 & 0 & 0 \\
0 & 506.6059 & 0 \\
0 & 0 & 506.6059
\end{array}\right) \quad\left(s^{2}\right) \\
\bar{K}^{-1} \bar{D}=\left(\begin{array}{rrr}
22.5045 & 0 & 0 \\
0 & 22.5045 & 0 \\
0 & 0 & 22.5045
\end{array}\right) \quad(s) .
\end{gathered}
$$

According to the Theorem, the closed-loop system is globally stable, and the system can track the commanded attitude.

Four simulations for the two wheel configurations are presented here. The first simulation results are shown in Figure 3 to Figure 5, which is Nadir pointing acquisition for the wheel aligned with body $y$ axis. The spacecraft points to the earth initially, but the attitude rate is zero. In order to point to the earth continuously, the pitch rate has 
to go to the orbit rate. Figure 3 shows that the attitude rate errors converge after about 300 seconds. The maximum attitude error during the transient is about 0.9 degrees. After the transient the steady attitude and rate errors are comparable with 3-axis wheel control

$$
\begin{aligned}
& \delta \phi=-7.9 \quad(\operatorname{arcsec}) \\
& \delta \theta=59 \quad(\operatorname{arcsec}) \\
& \delta \psi=-0.3 \quad(\operatorname{arcsec}) \\
& \Delta \omega_{x}=0.0003 \quad(\operatorname{arcsec} / s) \\
& \Delta \omega_{y}=0.00001 \quad(\operatorname{arcsec} / s) \\
& \Delta \omega_{z}=-0.01 \quad(\operatorname{arcsec} / s)
\end{aligned}
$$

The steady attitude errors are caused by the disturbance torques shown in Figure 5 . When the disturbance torques are not included in the simulation, the steady attitude errors are less than one arcseconds. In order to reduce the steady attitude errors, the gain matrices $D$ and $K$ in the control law can be increased, but they are constrained by considering additional spacecraft dynamics, such as flexible modes and fuel slosh. The magnetic dipoles usage and the wheel torque shown in Figure 4 are reasonable.

In the second simulation Nadir pointing with wheel along $\mathrm{z}$ axis is conducted as shown in Figure 6 and Figure 7. The spacecraft points to the earth and rotates with the orbit rate initially. The time period of the simulation is about two orbits. There are three times, in which the steady errors have transient. They are corresponding to $b_{z}(t)$ crossing zero three times. The absolute value of the minimum eigenvalue of $\Lambda_{z}(b(t))$ is used to measure the singularity. When the absolute value is less than 0.0002 , the inverse of $\Lambda_{z}(b(t))$ with the absolute value larger than 0.0002 is used to form the control law in the simulation. The singularity causes the transient, but the attitude errors transient is small and short. The control torques and the dipoles response significantly, but the maximum usage of the dipoles and the wheel torque are reasonable. The attitude control accuracy is comparable with 3-axis wheel control.

A pitch maneuver starting with Nadir pointing for the wheel aligned with body $y$ axis is conducted in the third simulation as shown in Figure 8 to Figure 10. The simulation starts with Nadir pointing attitude and rates. At 500 seconds the spacecraft begins to pitch up 90 degrees within 20 minutes and hold the 90 degrees pitch angle after the maneuver. During the maneuver the attitude and rate errors are small. The maximum pitch angle error is about 300 arcseconds. The dipoles and wheel torque usages are moderate. The command tracking during the maneuver is precise.

In the last simulation a yaw maneuver for the wheel aligned with body $y$ axis as shown in Figure 11 to Figure 13 is conducted. Different from the pitch maneuver, the Nadir pointing is preserved during the yaw maneuver. The yaw maneuver angle is 180 degrees with the duration of 40 minutes. The maneuver starts at 500 seconds. The attitude errors around about 2100 seconds have a spike, which is caused by $b_{y}(t)$ crossing zero. The maximum pitch angle error is about 1.8 degrees, but overall the yaw maneuver tracking is precise. Similar to the second simulation, the absolute value of the minimum eigenvalue of $\Lambda_{y}(b(t))$ is used to measure the singularity. When the absolute value is less than 0.0002 , the inverse of $\Lambda_{y}(b(t))$ with the absolute value larger than 0.0002 is used to form the control law in the simulation. Further the magnetic dipoles are limited within $\pm 400\left(A-m^{2}\right)$ and the wheel torque is limited within $\pm 0.02(\mathrm{Nm})$ to reflect the hardware limitation in real ACS design. The attitude and rate errors can be reduced with expansion of the limits for the magnetic dipoles and the wheel control torque. 

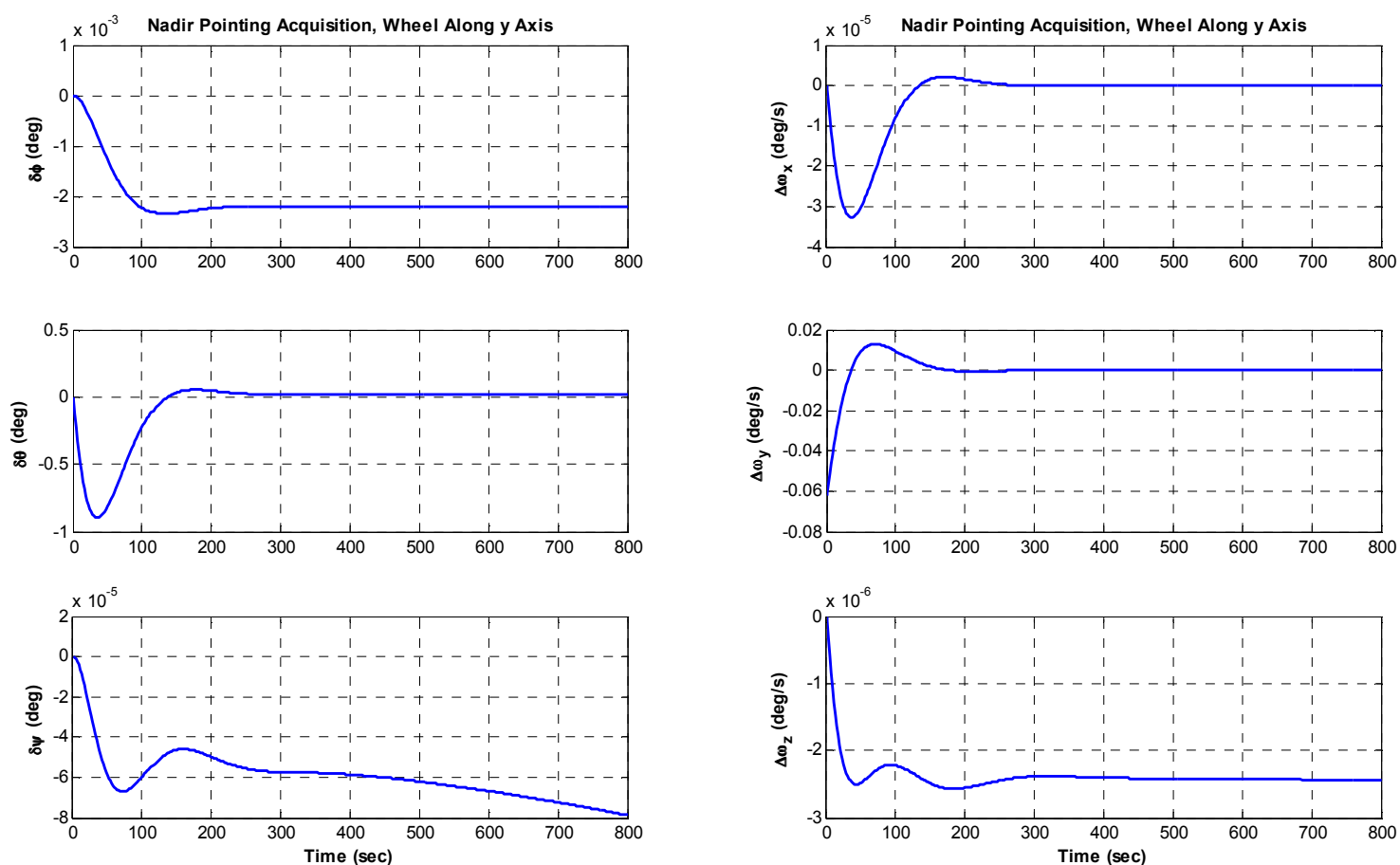

Figure 3 Attitude and rate errors during Nadir pointing acquisition
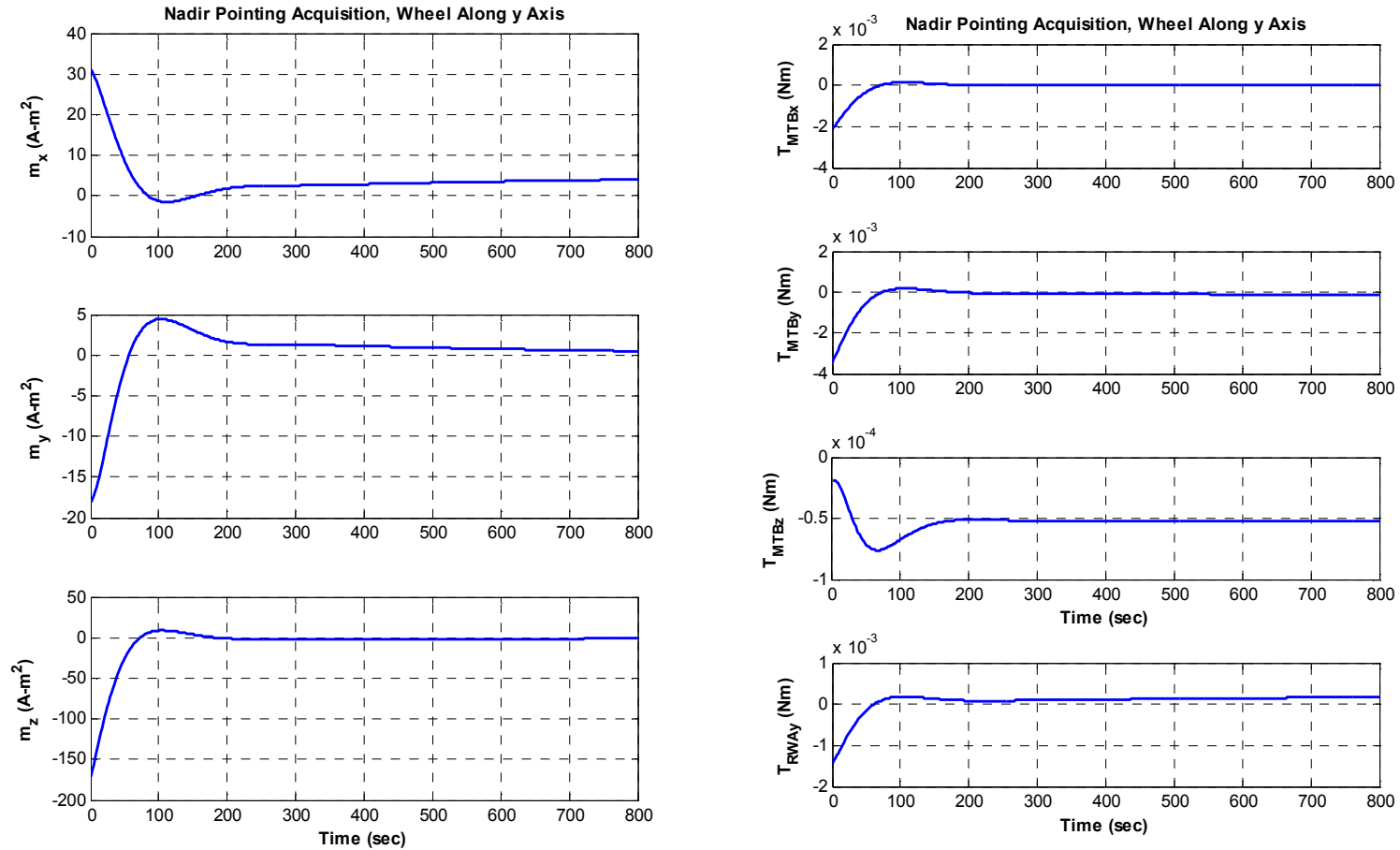

Figure 4 Magnetic dipoles and control torques during Nadir pointing acquisition 

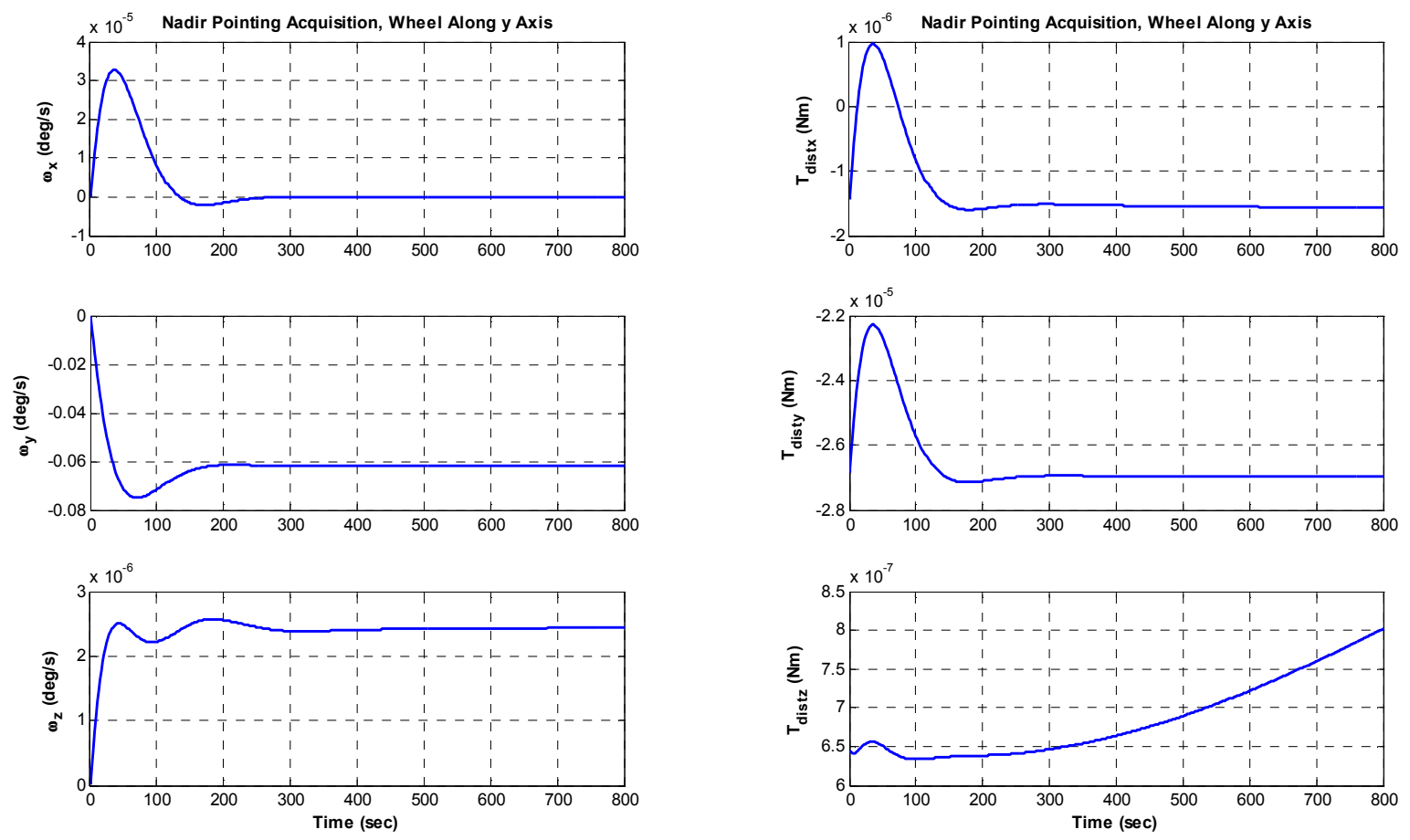

Figure $5 \mathrm{~S} / \mathrm{C}$ attitude rates and disturbance torques during Nadir pointing acquisition
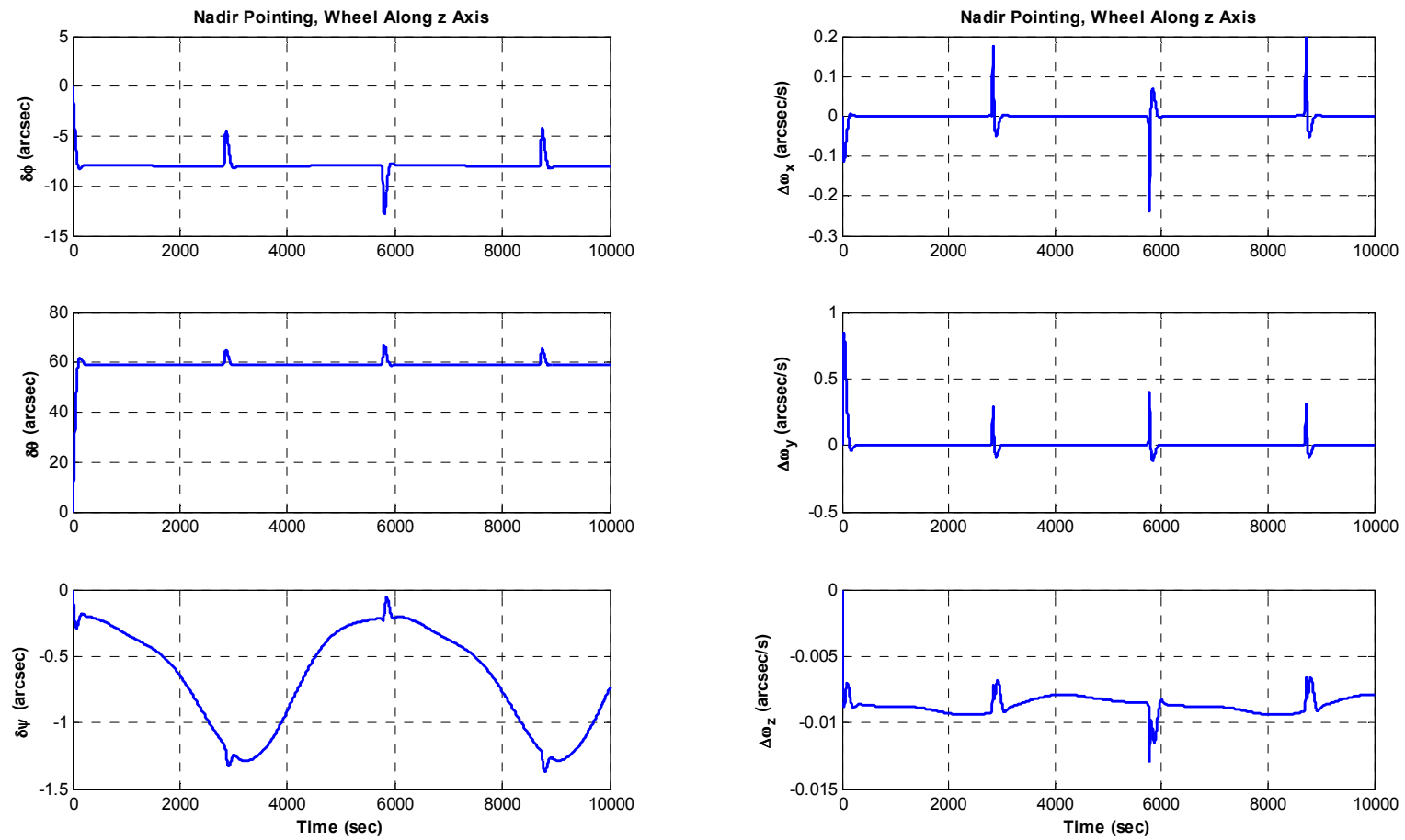

Figure 6 Attitude and rate errors during Nadir pointing with wheel along $\mathrm{z}$ axis 

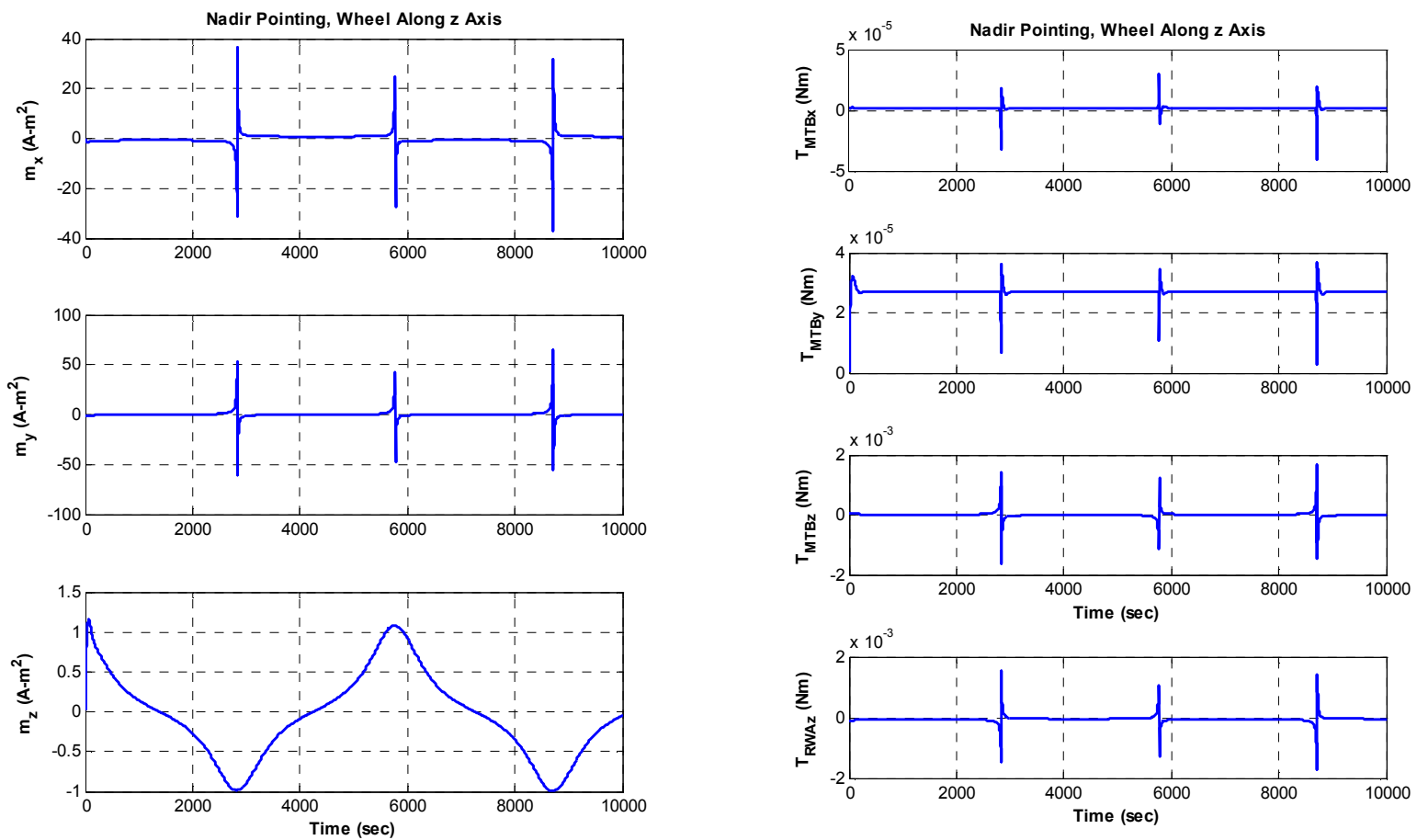

Figure 7 Magnetic dipoles and control torques during Nadir pointing with wheel along $\mathrm{z}$ axis
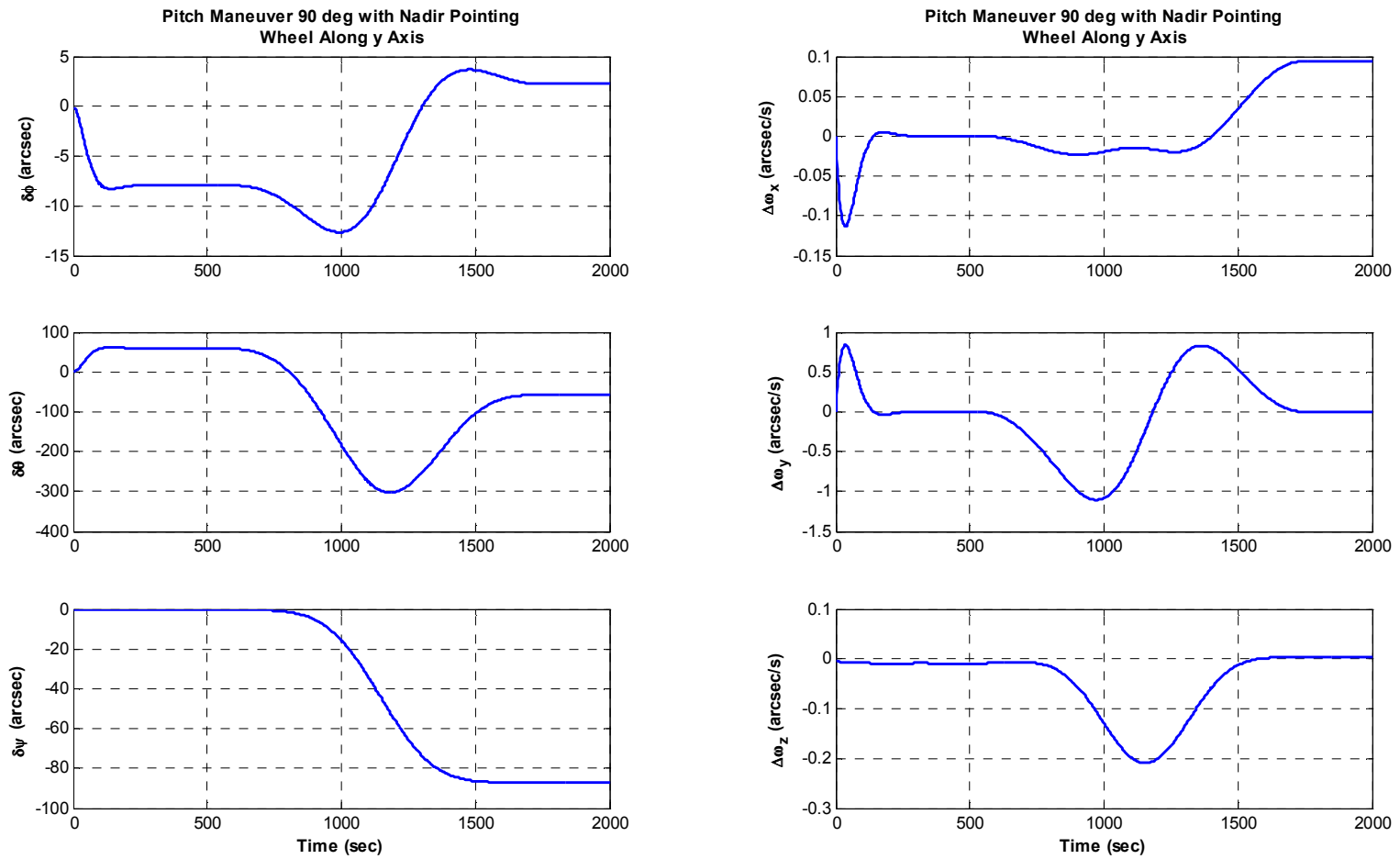

Figure 8 Attitude and rate errors during pitch maneuver with Nadir pointing 

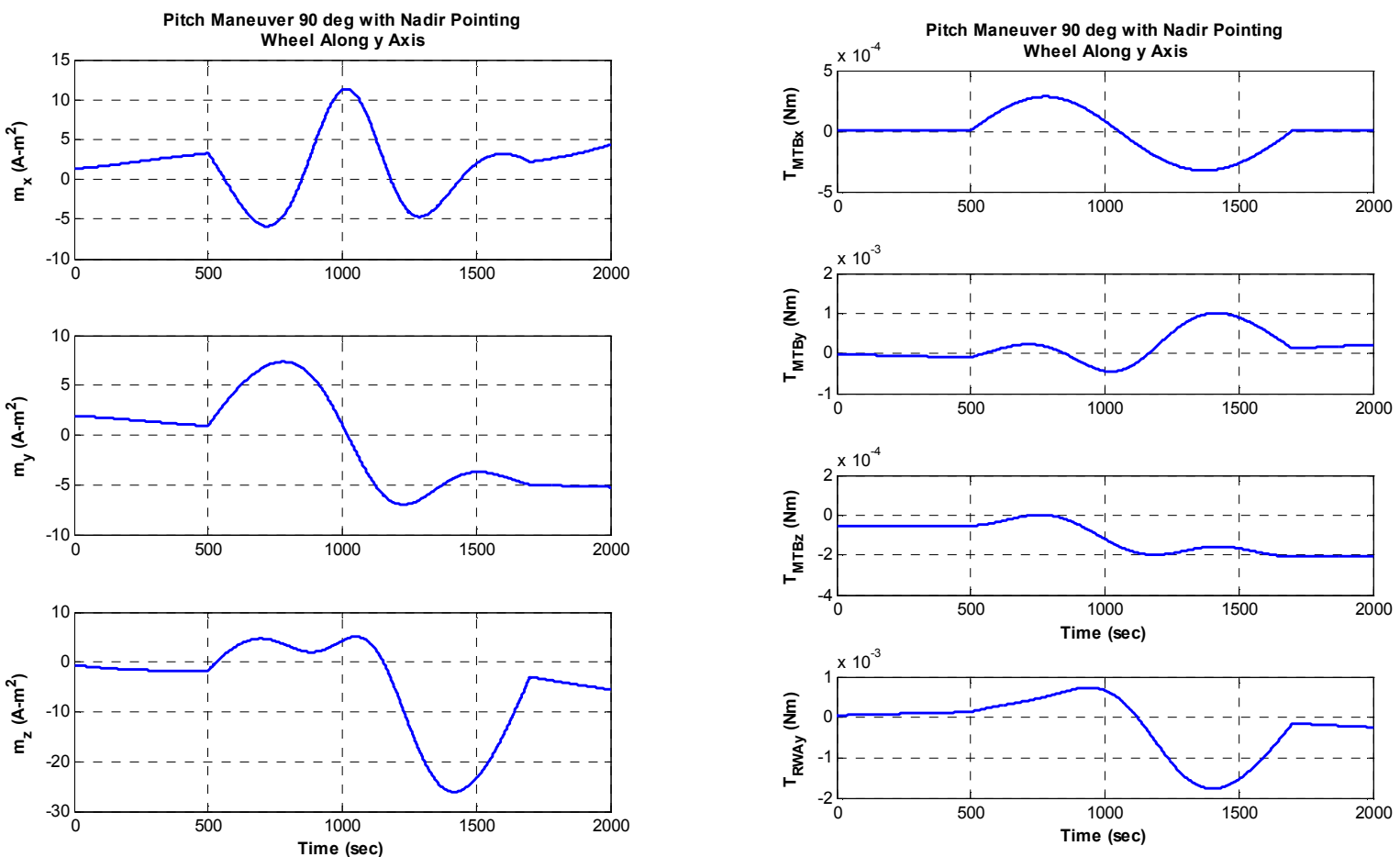

Figure 9 Magnetic dipoles and control torques during pitch maneuver with Nadir pointing
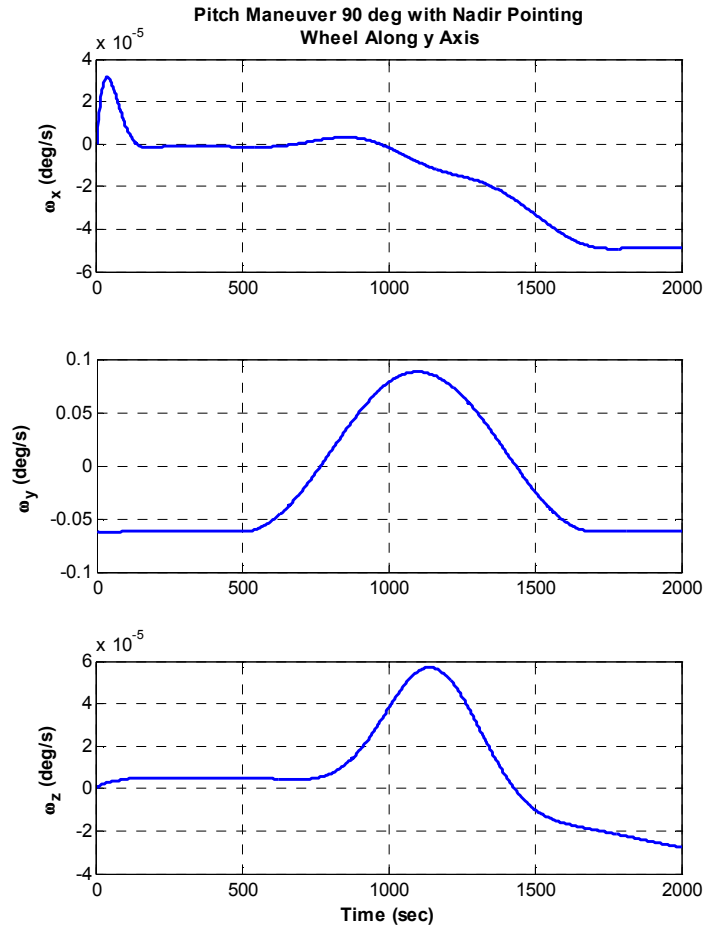

Figure $10 \mathrm{~S} / \mathrm{C}$ attitude rates during pitch maneuver with Nadir pointing 

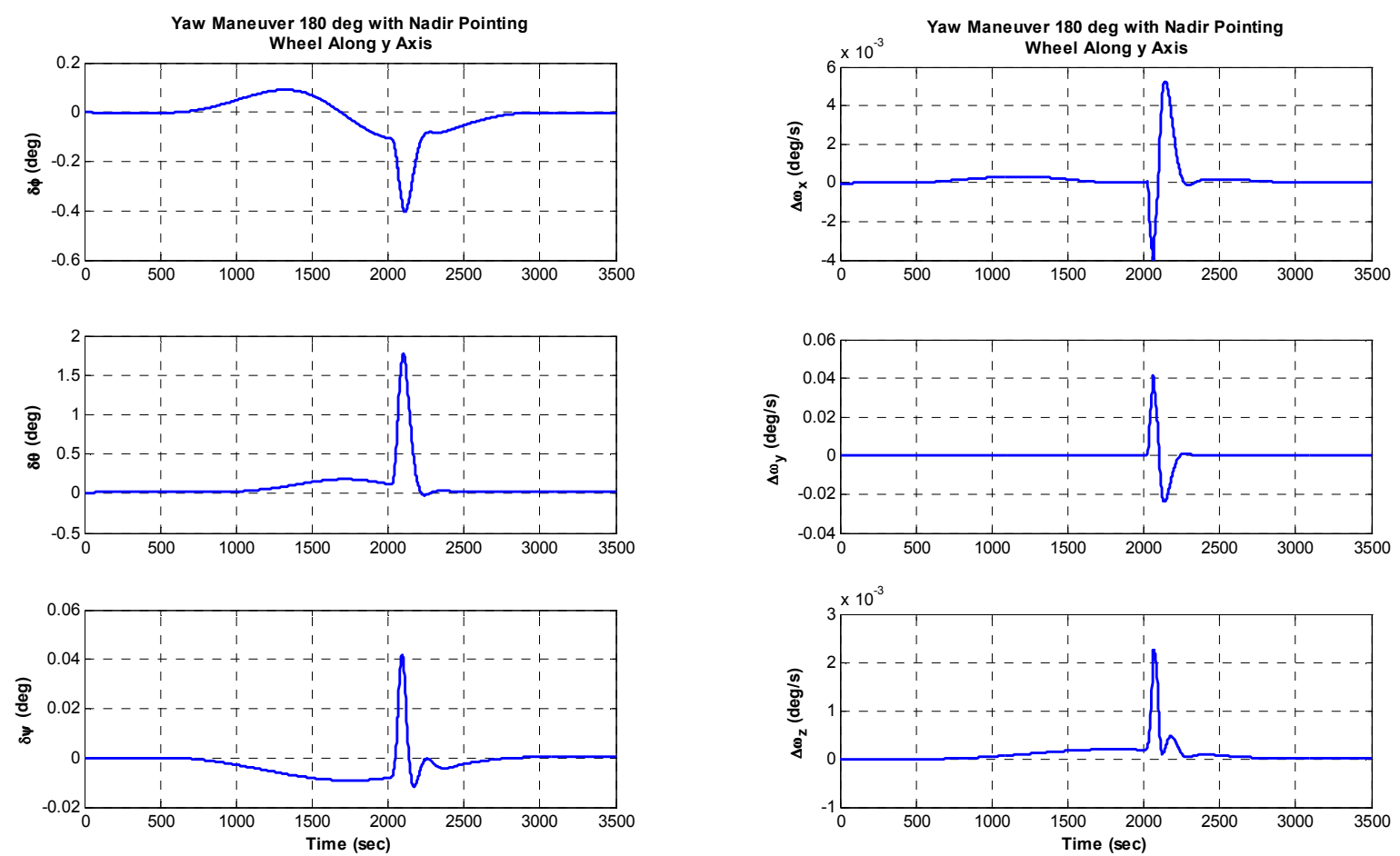

Figure 11 Attitude and rate errors during yaw maneuver with Nadir pointing
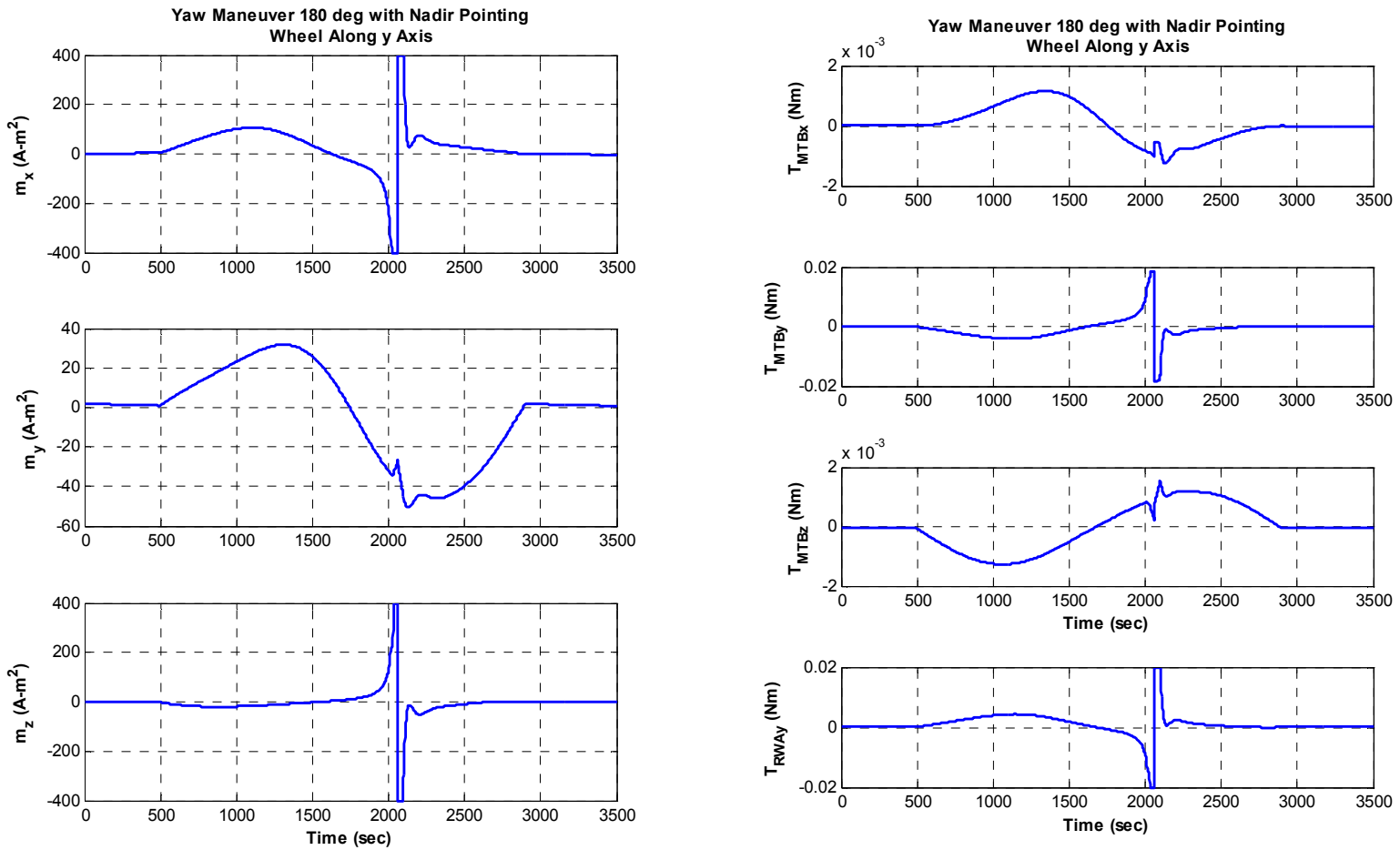

Figure 12 Magnetic dipoles and control torques during yaw maneuver with Nadir pointing 

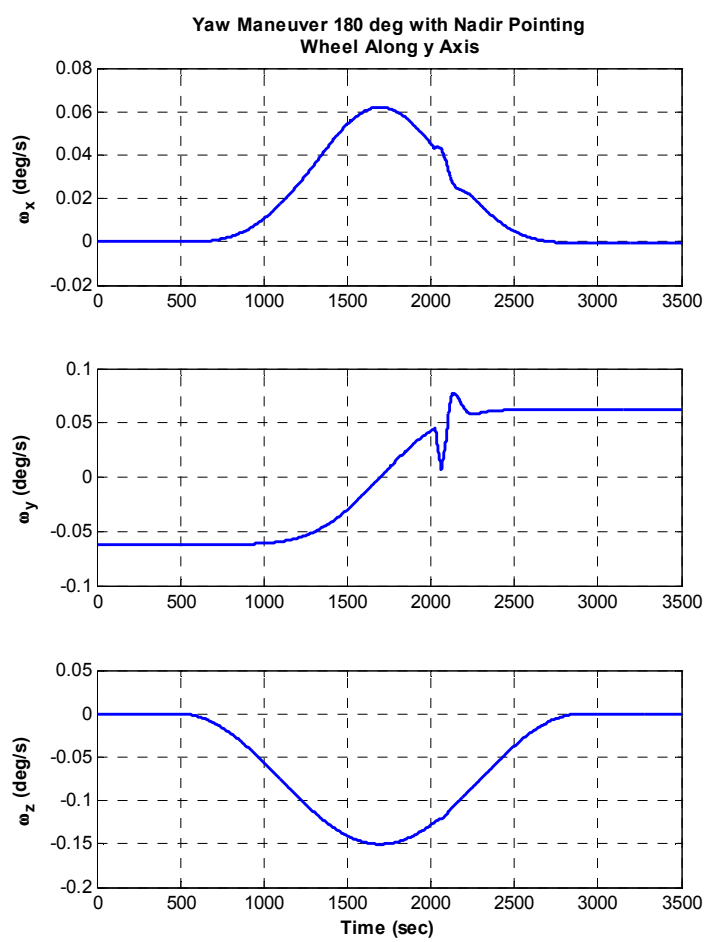

Figure $13 \mathrm{~S} / \mathrm{C}$ attitude rates during yaw maneuver with Nadir pointing

VII. Conclusions

Spacecraft attitude control using the combination of magnetic actuators with RWAs or other types of actuators, such as thrusters, is presented in this paper. The attitude control algorithms of the combined actuators are derived, which can guarantee the spacecraft attitude and rates to track the commanded values precisely. A design example is presented for Nadir pointing, 90 degrees pitch and 180 degrees yaw maneuvers. The results show that precise attitude tracking can be reached and the attitude control accuracy is comparable with 3-axis wheel control.

\section{Acknowledgments}

The author would like to thank Randy Regan for his support and encouragement.

\section{References}

${ }^{1}$ Lovera, M., and Astolfi, A., "Global magnetic attitude control of spacecraft in the presence of gravity gradient", IEEE Transactions on Aerospace and Electronic Systems, 42, 3 (2006).

${ }^{2}$ Lovera, M., and Astolfi, A., "Spacecraft attitude control using magnetic actuators", Automatica, 40, 8 (2004).

${ }^{3}$ Psiaki, M., "Magnetic torquer attitude control via asymptotic periodic linear quadratic regulation", Journal of Guidance, Control and Dynamics, 24, 2 (2001), 386-394.

${ }^{4}$ Yan, H., Ross, M., and Alfriend, K., "Pseudospectral feedback control for three-axis magnetic attitude stabilization in elliptic orbits", Journal of Guidance, Control and Dynamics, 30, 4 (2007).

${ }^{5}$ Wisniewski, R., "Linear time-varying approach to satellite attitude control using only electromagnetic actuation", Journal of Guidance, Control and Dynamics, 23, 4 (2000).

${ }^{6}$ Junkins, J., and Carrington, C., "Time-optimal magnetic attitude maneuvers", Journal of Guidance, Control and Dynamics, 4, 4 (1981).

${ }^{7}$ Wertz, J. R. (ed.), "Spacecraft Attitude Determination and Control", Kluwer Academic, Nowell, MA, (1978)

${ }^{8}$ Zhou, Z. and Colgren, R., "A Nonlinear Spacecraft Attitude Tracking Controller for Large Non-constant Rate Commands", International Journal of Control, 78, 5 (2005a).

${ }^{9}$ Zhou, Z. and Colgren, R., "Nonlinear Attitude Control for Large and Fast Maneuvers", AIAA-2005-6177, 2005 AIAA Guidance, Navigation, and Control Conference and Exhibit (2005b). 